\title{
Structural transitions in elemental tin at ultra high pressures up to $230 \mathrm{GPa}$
}

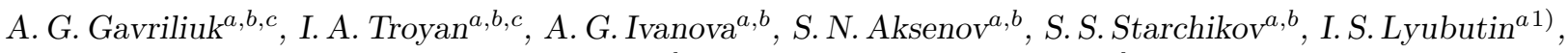 \\ W. Morgenroth ${ }^{d}$, K. V. Glazyrin ${ }^{e}$, M. Mezouar ${ }^{f}$ \\ ${ }^{a}$ Shubnikov Institute of Crystallography of FSRC "Crystallography and Photonics" RAS, 119333 Moscow, Russia \\ ${ }^{b}$ Institute for Nuclear Research, Russian Academy of Sciences, 108840 Troitsk, Russia \\ ${ }^{c}$ REC "Functional Nanomaterials", Immanuel Kant Baltic Federal University, 236041 Kaliningrad, Russia \\ ${ }^{d}$ Institut fur Geowissenschaften, Goethe-Universität, 60438 Frankfurt am Main, Germany \\ ${ }^{e}$ Deutsches Elektronen-Synchrotron DESY, Photon Science, 22607 Hamburg, Germany \\ ${ }^{f}$ European Synchrotron Radiation Facility, CS40220, F-38043 Grenoble, France \\ Submitted 31 October 2017 г.
}

\begin{abstract}
The crystal structure of elemental Sn was investigated by synchrotron X-ray diffraction at ultra high pressures up to $\sim 230 \mathrm{GPa}$ creating in diamond anvil cells. Above $70 \mathrm{GPa}$, a pure $b c c$ structure of Sn was observed, which is stable up to $160 \mathrm{GPa}$, until an occurrence of the $h c p$ phase was revealed. At the onset of the $b c c-h c p$ transition at pressure of about $160 \mathrm{GPa}$, the drop of the unit cell volume is about $1 \%$. A mixture of the $b c c-h c p$ states was observed at least up to $230 \mathrm{GPa}$, and it seems that this state could exist even up to higher pressures. The fractions of the $b c c$ and $h c p$ phases were evaluated in the pressure range of the phase coexistence 160-230 GPa. The difference between static and dynamic compression and its effect on the $V-P$ phase diagram of Sn are discussed.
\end{abstract}

DOI: 1234567890

Introduction. The search for high-temperature superconductivity is one of the major challenges in condensed matter physics and solid-state chemistry. The poly-hydrides of metals, including Sn, are promising candidates for superconductors, which can be obtained at very high pressures of the megabar range. Structural studies of Sn-hydrides using X-ray diffraction (XRD) are impossible without careful investigations of the structure of pure tin as a reference. Moreover, the ${ }^{119} \mathrm{Sn}$ Mössbauer isotope was recently used as a sensor of magnetic field in the search for superconductivity in $\mathrm{H}_{2} \mathrm{~S}$ compressed to $150 \mathrm{GPa}$ [1]. Therefore, the scientific community addresses the investigation of the tin structure in the multi-megabar pressure region as a very important fundamental problem.

There is a lot of literature related to theoretical and experimental studies of phase transitions and highpressure structural modifications of elemental Sn [2-14]. The crystal structure of white tin $\beta$-Sn (space group $I 41 /$ amd) at $9.8 \mathrm{GPa}$ and ambient temperature was shown to transform to body-centered tetragonal structure bct $I 4 / \mathrm{mmm}$ [15]. The pressure dependence of the

\footnotetext{
1) e-mail: lyubutinig@mail.ru
}

corresponding optical-phonon modes of metallic $\beta$-Sn has been studied up to $9.6 \mathrm{GPa}$ by Raman spectroscopy [16]. Desgreniers et al. [2] showed by X-ray study of tin up to $120 \mathrm{GPa}$ that at $45 \mathrm{GPa}$ a body-centered tetragonal $(b c t)$ structure transforms into a body-centered cubic $(b c c, I m-3 m)$ structure.

Salamat et al. [3] studied the structure of tin by angle-dispersive synchrotron X-ray diffraction up to $138 \mathrm{GPa}$ under quasi-hydrostatic conditions at room temperature. Their data confirm the occurrence of a first-order phase transition at $10.8 \mathrm{GPa}$ between $\beta$-Sn and a higher pressure phase, which is the body-centeredtetragonal polymorph $(b c t)$. Above $32 \mathrm{GPa}$, this phase exhibits a distortion into the body-centered orthorhombic (bco, Immm) modification. Beyond $70 \mathrm{GPa}$, the structure undergoes another phase transition and becomes body-centered cubic $(b c c)$. In the pressure range of 40-70 GPa, the coexistence of the bcc and bco structures was observed [3]. Salamat et al. [4] reported the first experimental observation of the hexagonal closepacked $\left(h c p, P 6_{3} / m m c\right)$ phase that evolved from the $b c c$ structure at $157 \mathrm{GPa}$ and both phases coexisted up to $194 \mathrm{GPa}$ (see also Fig. 1). Such a martensitic transition is not new for high pressure behavior and was reported 

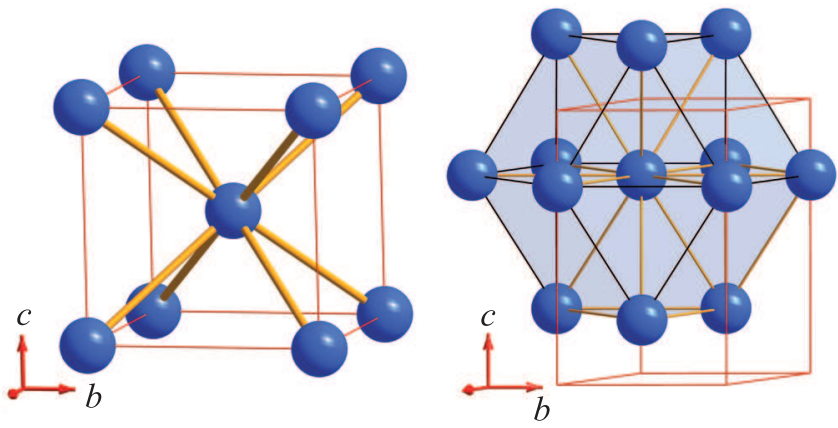

Fig. 1. (Color online) The unit cells of bcc and hcp structures of Sn. These two structures coexist in the pressure range 160-230 GPa. Both structures have the same number of atoms per unit cell

for many materials, including pure iron. Usually, similar transitions are related to a strong modification of electronic properties making the pure tin additionally attractive as an object of solid state research.

In our study, the crystal structure of Sn was investigated by synchrotron XRD technique at high pressures up to a maximal value of $\sim 230 \mathrm{GPa}$ creating in diamond anvil cells and using different high pressure transmitting media. We revealed the onset of a phase transition from Sn-bcc to Sn- $h c p$ structure at a pressure of about $160 \mathrm{GPa}$ similar to the results obtained previously [4]. Both phases coexist in the broad pressure range of 160 $230 \mathrm{GPa}$ despite the softest pressure media used in this work.

Experiments. The tin foil samples enriched with the ${ }^{119} \mathrm{Sn}$ Mössbauer isotope up to $95 \%$ was used in our study. Synchrotron XRD studies of structural properties were performed at high pressures in the diamond anvil cell (DAC) in different pressure media $\left(\mathrm{He}, \mathrm{H}_{2}\right.$, silicon oil PES-5, $\mathrm{NaCl}$ ) at room temperature. Experiments were performed at beam-line ID27 of ESRF (Grenoble, France) [17] and at beam-line P02.2 of PETRA III at DESY [18].

Five runs of the XRD measurements were performed. At maximal pressures we used DAC design similar to [19]. The tin foil samples were placed into the volume of the hole in the gasket of the DAC chamber. The gasket materials in different runs were Re or ceramic made of the X-ray amorphous composite mixture of $\mathrm{CaF}_{2}$ with epoxy. The beveled shape of diamond anvils with typical diameter of flat culets from $40 \mu \mathrm{m}$ to $70 \mu \mathrm{m}$ was used in different runs. The typical diameter of the gasket hole varied from $15 \mu \mathrm{m}$ to $25 \mu \mathrm{m}$. The sample was prepared from a metal ${ }^{119} \mathrm{Sn}$ foil by squeezing it to a thickness of $\sim 2-3 \mu \mathrm{m}$. The pressure was measured using the diamond Raman scale [20] and in some runs it was obtained from the X-ray diffraction of $\mathrm{NaCl}$ (B2 phase) [21] and of $\mathrm{Au}$ [21]. The typical images of sample chambers are shown in Fig. 2.
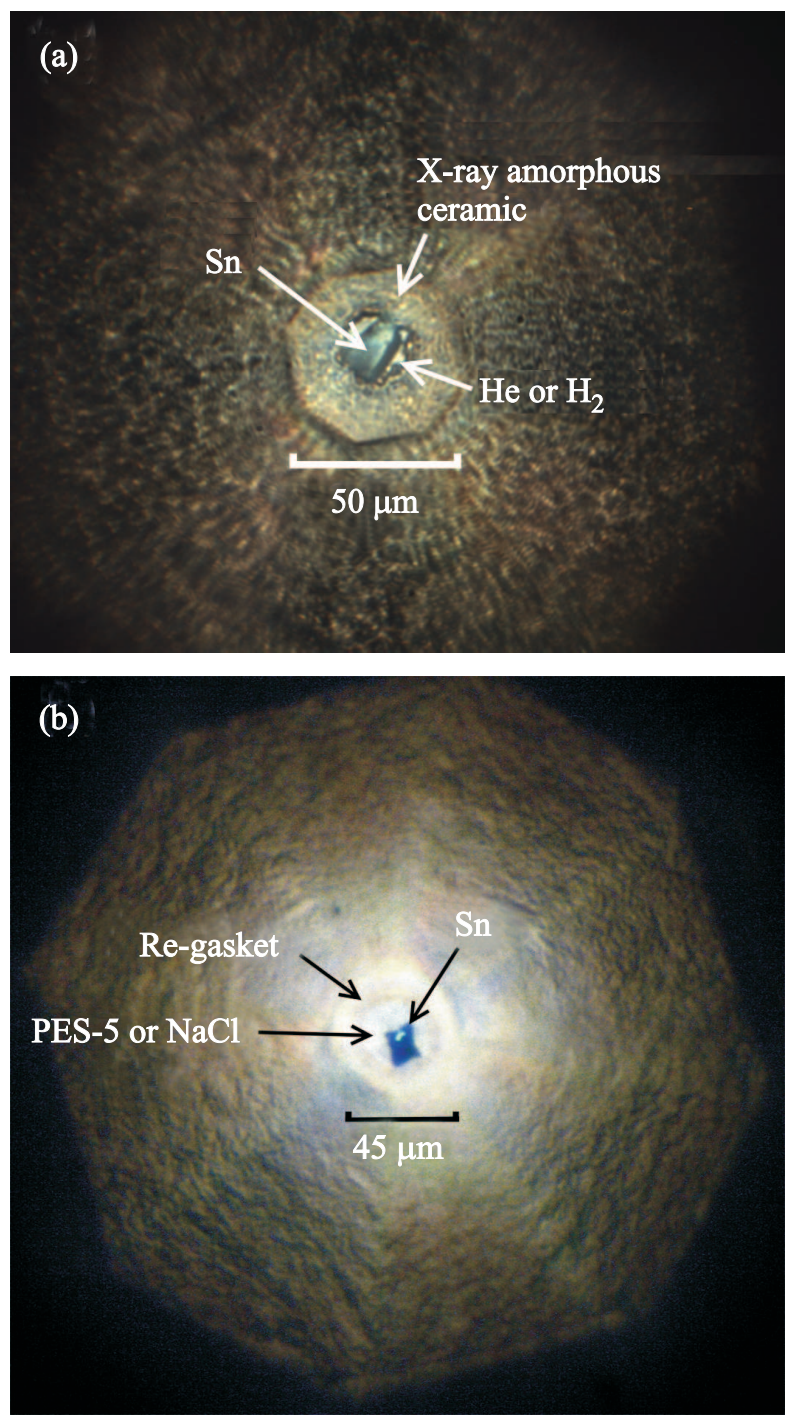

Fig. 2. (Color online) The typical images of experimental mounts: (a) - The gasket material is $\mathrm{CaF}_{2}$ ceramic; $\mathrm{H}_{2}$ or He was used as pressure medium; culet diameter is $\sim 50 \mu \mathrm{m}$; bevel diameter is $\sim 300 \mu \mathrm{m}$. (b) - The gasket material is Re; silicon oil PES-5 or $\mathrm{NaCl}$ was used as a pressure transmitting medium; cullet diameter is $\sim 45 \mu \mathrm{m}$; bevel diameter is $\sim 240 \mu \mathrm{m}$. The images were obtained using transmitting and reflected light setting, simultaneously. In the gasket holes, chips of tin foil surrounded by transparent pressure media can be clearly seen

At the ESRF facilities, the XRD patterns were recorded using monochromatic X-ray radiation $(\lambda=$ $=0.3738 \AA$, spot size of $3 \mu \mathrm{m}$ ) with a MarCCD detector at working distance of $192 \mathrm{~mm}$. At PETRA-III we used wavelengths $\lambda=0.2907 \AA$ and $\lambda=0.2945 \AA$ in different 
runs. The spot size at the sample was $2 \times 2.2 \mu \mathrm{m}$. A two-dimensional area (flat panel) Perkin Elmer (XRD 1621) detector was set at a distance of 462.92 or at $491 \mathrm{~mm}$ from the sample, depending on a run. Diffraction data were integrated using DIOPTAS program [22]. The diffraction patterns were fitted by the Le Bail method using program JANA2006 [23].

Results and discussion. The high-pressure evolutions of synchrotron XRD patterns of tin in helium and in hydrogen media are shown in Fig. 3. As expected, the

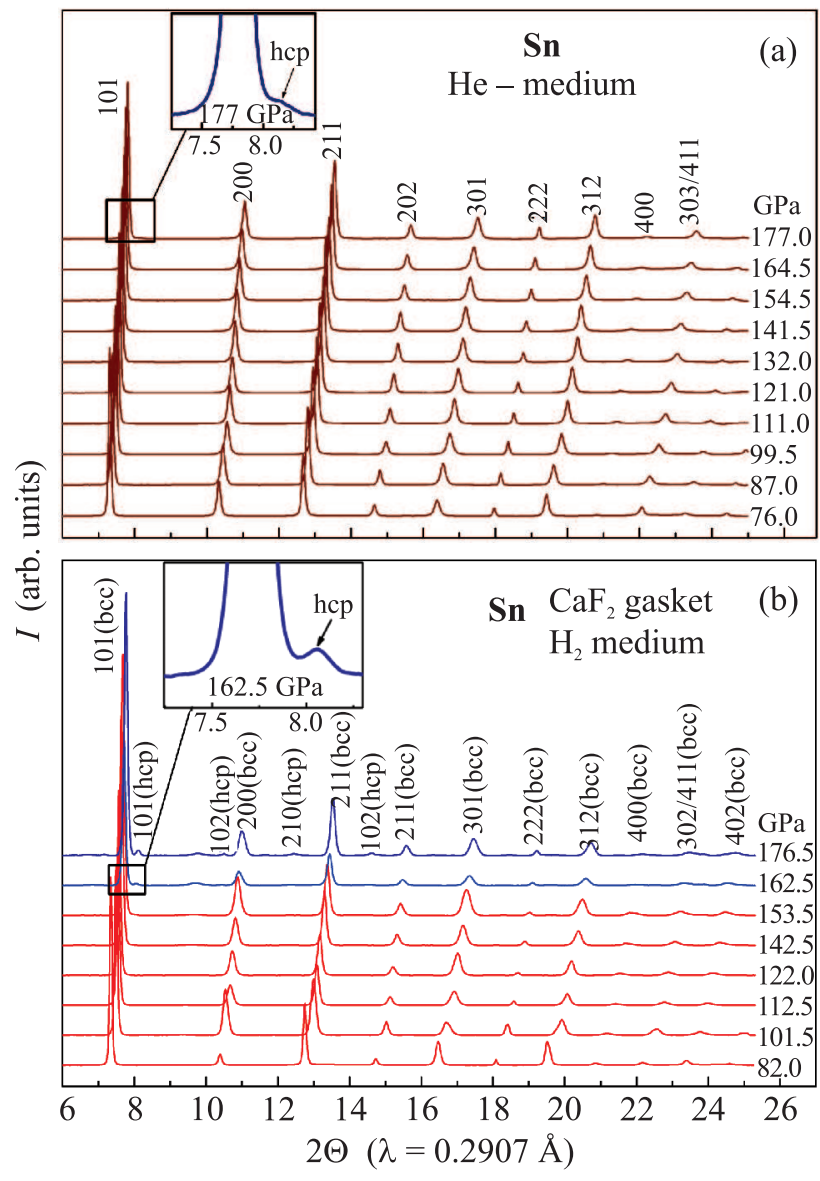

Fig. 3. (Color online) The evolution of room-temperature XRD patterns of tin at compression up to $\sim 177 \mathrm{GPa}$. (a) - Helium is the pressure medium. The Sn-bcc reflex indexes are indicated. Inset shows the Sn-hcp 101 reflection at $177 \mathrm{GPa}$ in an enlarged scale. (b) - Hydrogen is the pressure medium. Inset shows the Sn-hcp 101 reflection at $162.5 \mathrm{GPa}$ in an enlarged scale. Red curves show XRD patterns before the onset of the $b c c-h c p$ phase transition

diffraction peaks shift to higher angles with increasing pressure. The measurements were analysed in the angle range of $2 \Theta \leq 28^{\circ}$. Unit cell parameters were determined from Le Bail fitting by JANA2006 [23].
In the measurements with He pressure medium we observed a very weak peak to the right of the 110 reflection of Sn-bcc (inset in Fig. 3a), which can indicate the appearance of the hcp phase at the maximal pressure in this run to $177 \mathrm{GPa}$.

Hydrogen and helium are considered the softest pressure media known today; therefore, these results are the most reliable from the point of view of quasi-hydrostatic conditions. However, the complexity of the experiment and geometry of compression can have its influence. We estimated that the onset of the bcc-hcp transition in $\mathrm{Sn}$, observed at the most hydrostatic conditions of helium, starts at $\sim 177 \pm 5 \mathrm{GPa}$ (inset in Fig. 3a). The error of $\pm 5 \mathrm{GPa}$ in the accuracy of pressure determination was evaluated from the accuracy of a diamond Raman scale in our experiment.

In Fig. $3 \mathrm{~b}$ and Fig. 4a, the evolutions of XRD patterns of $\mathrm{Sn}$ in $\mathrm{CaF}_{2}$ gasket material are shown in the presence of hydrogen. Here, it is possible that a small amount of $\mathrm{H}_{2}$ was present around the sample and inside the gasket material near the hole. Initially, $\mathrm{H}_{2}$ was filled inside the gasket hole together with Sn chips, but with increasing pressure, the hydrogen medium disappeared almost completely. We suppose that hydrogen was partly blown out and partly absorbed by tin, but only in the amount insufficient to synthesize a stoichiometric tin hydride. In this case hydrogen atoms probably form irregular interstitial defects.

In such a complex environment, we observed the onset of the bcc-hcp transition which was detected by appearance of Sn-hcp 101 reflection at $\sim 162 \mathrm{GPa}$ (inset in Fig. 3b) and at $\sim 158 \mathrm{GPa}$ (Fig. $4 \mathrm{a}$ ).

The evolution of XRD patterns of $\mathrm{Sn}$ in $\mathrm{NaCl}$ pressure medium is shown in Fig. $4 \mathrm{~b}$. The onset of the $b c c-$ hcp phase transition was observed between 156 and $163 \mathrm{GPa}$.

In the DAC cell with the PES-5 pressure media, the XRD measurements at pressures up to $197 \mathrm{GPa}$ were performed on the ESRF facilities with a wavelength of $0.3738 \AA$, whereas the measurements at above $200 \mathrm{GPa}$ were made on PETRA-III with a wavelength of $0.29045 \AA$. To reconcile and compare the results of these measurements, the evolution of diffraction patterns of $\mathrm{Sn}$ is given in Fig. 4c as a function of the backscattering vector $Q$. In this case, the onset of the bcc-hcp transition was observed between 148 and $169 \mathrm{GPa}$. The estimated values of pressures of the $b c c-h c p$ transition of Sn in various transmitting media are in agreement with data of Ref. [4].

The full set of our experimental XRD data was carefully analysed and we calculated phase composition and $V-P$ equation of state (EOS) of $\mathrm{Sn}$ at room tempera- 

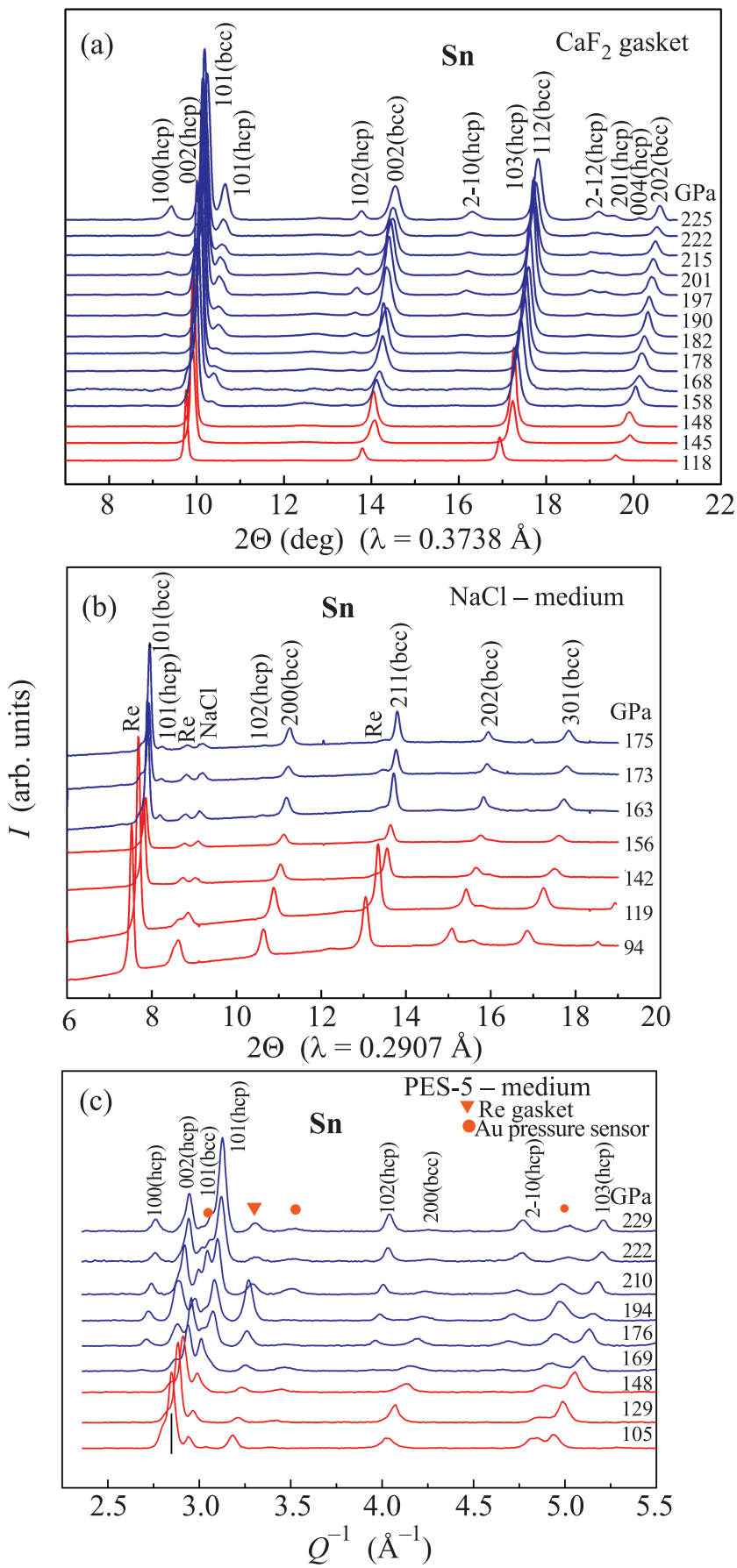

Fig. 4. (Color online) The evolution of room-temperature $\mathrm{XRD}$ patterns of tin upon compression in various pressure media: (a) - in $\mathrm{NaCl}$ up to $175 \mathrm{GPa}$, (b) - in a $\mathrm{CaF}_{2}$ gasket environment with possibly a small amount of hydrogen $\left(\mathrm{H}_{2}\right)$ up to $\sim 225 \mathrm{GPa},(\mathrm{c})$ - in PES-5 up to $229 \mathrm{GPa}$. Peak positions for reflections of $h c p$ and $b c c$ structure are indicated. Red curves show XRD patterns before the onset of the $b c c-h c p$ phase transition. Orange triangles and cycles indicate $\mathrm{Re}$ and $\mathrm{Au}$ reflections, respectively ture in the pressure region of $76-230 \mathrm{GPa}$. At pressures higher than the onset of the $b c c-h c p$ transition we observed and documented the clear evidence of coexistence of the $b c c$ and the $h c p$ phases. The measured $V-P$ relations of $\mathrm{Sn}$ for the $b c c$ and the $h c p$ phases are shown in Fig. 5. The unit cell volumes obtained experimentally

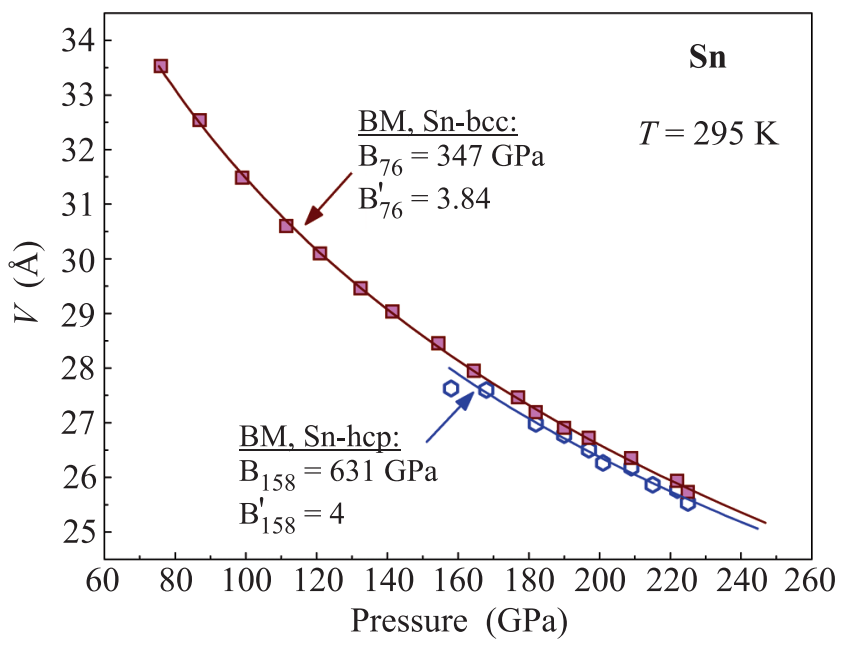

Fig. 5. (Color online) The room temperature EOS of Sn for the volume of the $b c c$ and $h c p$ phases at pressure increase from 76 to $230 \mathrm{GPa}$ calculated from the set of XRD measurements with various pressure transmitting media. The symbols are the experimental points. The lines show the fits using a modified $2^{\text {nd }}$ order Birch-Murnaghan equation of state. At pressures higher than the onset of the bcc-hcp transition the $b c c$ and the $h c p$ phases coexist

fit well to the modified Birch-Murnaghan EOS:

$$
\begin{aligned}
P- & P_{n}=\frac{3}{2} B_{n}\left(\frac{V}{V_{n}}\right)^{-\frac{5}{3}}\left(1-\left(\frac{V}{V_{n}}\right)^{-\frac{2}{3}}\right) \times \\
\times & {\left[\frac{3}{4}\left(B_{n}^{\prime}-4\right)\left(1-\left(\frac{V}{V_{n}}\right)^{-\frac{2}{3}}\right)-1\right], }
\end{aligned}
$$

where $P_{n}$ is the evaluated pressure of the transition into the current high-pressure phase, $V_{n}$ is the unit cell volume at pressure $P_{n}, B_{n}$ is the bulk modulus, and $B_{n}^{\prime}$ is the pressure derivative of $B_{n}$. For the Sn$b c c$ phase, the obtained parameters are: $P_{76}=76 \mathrm{GPa}$, $V_{76}=33.46 \pm 0.08 \AA^{3}, B_{76}=347 \pm 13 \mathrm{GPa}, B_{76}^{\prime}=$ $=3.8 \pm 0.2$. For the Sn-hcp phase, the obtained parameters are: $P_{158}=158 \mathrm{GPa}, V_{158}=27.77 \pm 0.09 \AA^{3}$, $B_{158}=631 \pm 15 \mathrm{GPa}, B_{158}^{\prime}=4$ (fixed value). The difference in the cell volumes between the bcc and hcp phases at $\sim 168 \mathrm{GPa}$ is about $1.0 \%$.

The content (fraction) of the hcp phase obtained from the quantitative Rietveld analysis increases with increasing pressure. The behavior of the content of the 
hcp phase observed at pressure increase in silicon oil PES-5 and in $\mathrm{CaF}_{2}$ pressure media is shown in Fig. 6. In the PES-5 medium, the hcp fraction grows approxi-

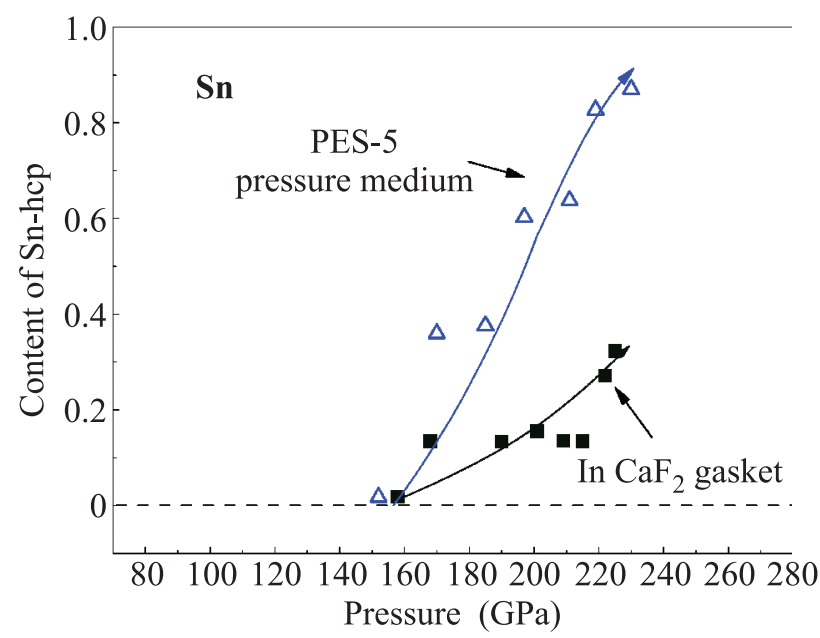

Fig. 6. (Color online) The increase of the hcp phase content upon compression in silicon oil (PES-5) pressure medium and in $\mathrm{CaF}_{2}$ gasket environment. In the $\mathrm{CaF}_{2}$ gasket environment possibly was a small amount of hydrogen (see text for details). In the $\mathrm{CaF}_{2}$ gasket environment a small amount of hydrogen can possibly be present (see text for details). Solid lines are guides for the eye

mately with the same rate as it was observed in the $\mathrm{Ne}$ pressure medium [4]. We found that in silicon oil the $h c p$ fraction grows up to $90 \%$ at $229 \mathrm{GPa}$. In the $\mathrm{CaF}_{2}$ gasket environment (with possible presence of a small amount of hydrogen), the content of the hcp phase grows much weaker than in the other pressure media (e.g. see Fig. 6). We evaluated that in this case the fraction of the hcp phase increases only to $30 \%$ at $225 \mathrm{GPa}$.

The relatively small difference in the unit cell volume of the $b c c$ and the $h c p$ phases together with our observation of compression behavior in different "solid" pressure media indicates that the $b c c-h c p$ transition may be strongly sensitive to the degree of hydrostaticity and to the influence of undesirable stresses, i.e. microstrains.

The obtained results are important for a comprehensive understanding of Sn as a complex electronic system characterized by multiple phase transitions induced by high pressure.

Summary. The crystal structure of elemental Sn was investigated at ultra pressures up to $\sim 230 \mathrm{GPa}$ at room temperature. We started the measurements at $\sim 70 \mathrm{GPa}$ and found pure bcc phase of Sn above $70 \mathrm{GPa}$ in complete agreement with results observed previously $[2,3,5]$. Upon further compression, the onset of transition into the hcp phase at $\sim 160 \mathrm{GPa}$ was observed. This is the first experimental acknowledgement up to date of the appearance of the hcp phase previously observed in [4]. Thus, our results confirm principal difference between static [4] and dynamic [14] compression on the phase diagram of Sn. At static compression, the $b c c$ phase lost stability and transforms into a complicate mixture of $b c c$ and $h c p$ phases at pressures about $160 \mathrm{GPa}$, whereas at dynamic compression [14], the bcc phase is stable up to $1.2 \mathrm{TPa}$ and there is no evidence of the $h c p$ phase. In our experiments, the mixed of the $b c c-h c p$ states was observed at least up to $230 \mathrm{GPa}$, and it looks like this state could exist even up to higher pressures.

In addition to the first observation of the hcp phase [4] in the pressure range of 157-194 GPa, we established that the range of pressures of the coexistence of the $b c c$ $h c p$ phases increases up to $\sim 230 \mathrm{GPa}$. The influence of pressure and pressure medium on the $h c p$-phase content was also revealed and documented.

It was evaluated that in the silicon oil pressure medium, the $h c p$ fraction grows up to $90 \%$ at $229 \mathrm{GPa}$, whereas in $\mathrm{CaF}_{2}$ medium with a possible small amount of hydrogen it only grows to $30 \%$ at $225 \mathrm{GPa}$. The lower content of the $h c p$-phase in the later case may be the result of the influence of small amount of hydrogen penetrating to the Sn sample. Our observations show that at high pressure the sample is broken into grains, whose size decreases with increasing pressure. Most probably, the nucleation of a new phase occurs within the grain boundaries. The fraction of intergrain boundaries increases with pressure, thus increasing the volume of the Sn-hcp phase. Hydrogen penetrates most easily and deeper into the volume of metal along grain boundaries, and thus Sn-hydrides can be created. These interesting effects should be investigated in further studies.

We are grateful to Dr. Hanns-Peter Liermann for assistance at the P02.2 beamline of DESY (PETRA III, Hamburg, Germany). We thank Dr. V.Struzhkin for reading the manuscript and providing his comments and corrections. The work was performed under support of Russian Ministry of Science and Education contract \# 14.616.21.0068. WM acknowledges BMBF project $05 \mathrm{~K} 13 \mathrm{RF} 1$. At preparation of sample mounts, the facilities of Center for Collective Use "Accelerator Center for Neutron Research of the Structure of Substance and Nuclear Medicine" of the INR RAS were used.

1. I. A. Troyan, A. G. Gavriliuk, R. Rüffer, A. Chumakov, A. A. Mironovich, I. S. Lyubutin, D. Perekalin, A. Drozdov, and M. Eremets, Science 351, 1303 (2016).

2. S. Desgreniers, Y. K. Vohra, and A. L. Ruoff, Phys. Rev. B 39, 10359 (1988). 
3. A. Salamat, R. Briggs, P. Bouvier, S. Petitgirard, A. Dewaele, M. E. Cutler, F. Cor‘a, D. Daisenberger, G. Garbarino, and P.F. McMillan, Phys. Rev. B 88, 104104 (2013).

4. A. Salamat, G. Garbarino, A. Dewaele, P. Bouvier, S. Petitgirard, C. J. Pickard, P.F. McMillan, and M. Mezouar, Phys. Rev. B 2011, 140104(R) (2011).

5. Q.-M. Jing, Y.-H. Cao, Y. Zhang, Sh.-R. Li, Q.Y.H. He, Sh.-G. Liu, L. Liu, Y. Bi, H.-Y. Geng, and Q. Wu, Chin. Phys. B 25, 120702 (2016).

6. B. H. Cheong and K. J. Chang, Phys. Rev. B 44, 4103 (1991).

7. N.E. Christensen and M. Methfessel, Phys. Rev. B 48, 5797 (1993)

8. A. Aguado, Phys. Rev. B 67, 212104 (2003).

9. Ch. Yu, J. Liu, H. Lu, and J. Chen, Sol. State Comm. 140, 538 (2006).

10. R. G. McQueen and S. P. Marsh, J. Appl. Phys. 31, 1253 (1960).

11. K. V. Khishchenko, J. Phys.: Conf. Series 121, 022025 (2008).

12. Y. Yao and D. D. Klug, Sol. State Comm. 151, 1899 (2011)

13. D. Mukherjee, K. D. Joshi, and S. C. Gupta, J. Phys.: Conf. Series 215, 012106 (2010).
14. A. Lazicki, J.R. Rygg, F. Coppari, R. Smith, D. Fratanduono, R. G. Kraus, G. W. Collins, R. Briggs, D. G. Braun, D. C. Swift, and J. H. Eggert, Phys. Rev. B 115, 075502 (2015).

15. J. D. Barnett, V. E. Bean, and H. T. Hall, J. Appl. Phys. 37, 875 (1966).

16. H. Olijnyk, Phys. Rev. B 46, 6589 (1992).

17. M. Mezouar, W.A. Crichton, S. Bauchau, F. Thurel, H. Witsch, F. Torrecillas, G. Blattmann, P. Marion, Y. Dabin, J. Chavanne, O. Hignette, C. Morawe, and C. Borel, J. Synchrotron Rad. 12 (2005) 659.

18. H.-P. Liermann, W. Morgenroth, A. Ehnes, A. Berghauser, B. Winkler, H. Franz, and E. Weckert, J. Phys. Conf. Ser. 215, 012029 (2010).

19. A. G. Gavriliuk, A. A. Mironovich, and V. V. Struzhkin, Rev. Sci. Instrum. 80, 043906 (2009).

20. Y. Akahama and H. Kawamura, J. Appl. Phys. 100, 043516 (2006).

21. Y. Fei, A. Ricolleau, M. Frank, K. Mibe, G. Shen, and V. Prakapenka, PNAS 104, 9182 (2007).

22. C. Prescher and V.B. Prakapenka, High Pressure Research 35, 223 (2015)

23. V. Petricek, M. Dusek, and L. Palatinus, Z. Kristallogr. 229, 345 (2014). 